\title{
THE AMBIPOLAR FILAMENTATION IN THE WARM IONIZED MEDIUM
}

\author{
M. FRANQUEIRA ${ }^{1}$, M. TAGGER ${ }^{2}$ and A.I. GÓMEZ DE CASTRO ${ }^{1}$ \\ ${ }^{1}$ Sec. Dept. Astronomía y Geodesia, Fac. CC. Matemáticas, Universidad Complutense de Madrid, \\ Spain;E-mail:mf@mat.ucm.es; merchefr@terra.es \\ ${ }^{2}$ Service d'Astrophysique, DSM/DAPNIA, C.E.A. Saclay
}

\begin{abstract}
We present the 2-D, two fluid (ions + neutrals) numerical simulations that we are carrying out in order to study the ambipolar filamentation process, in which a magnetized, partially ionized plasma is stirred by turbulence in the ambipolar frequency range. The higher turbulent velocity of the neutrals in the most ionized regions gives rise to a non-linear force driving them out of these regions, and causes the ions and the magnetic flux to condense in the most ionized regions, resulting in a filamentary structure where initial ionization inhomogeneities are amplified. This mechanism might help to explain some features observed in magnetized and partially ionized astrophysical plasmas as the interstellar medium.
\end{abstract}

Keywords: magnetohydrodynamics, turbulence, ISM: magnetic fields, numerical methods

\section{Introduction}

Kulsrud and Pearce (1969) and McIvor (1977) showed that in the magnetized and weakly ionized interstellar medium hydromagnetic waves are heavily damped in a frequency range between ion-neutral $\left(v_{\text {in }}\right)$ and neutral-ion $\left(v_{\text {ni }}\right)$ collision frequencies. In this ambipolar range neutrals and ions are imperfectly coupled. The ion-neutral friction causes, on one hand, energy dissipation, which results in the heating of the material (Piddington, 1956; Mestel and Spitzer, 1956) and, on the other hand, momentum transfer, that give rise to an acceleration of the material in the direction of propagation of the wave (Haerendel, 1992). As a consequence waves are strongly damped.

The non-linear evolution of this process can cause the ambipolar filamentation of the magnetic field (Tagger et al., 1995). When a magnetized and weakly ionized plasma showing small variations in the ionization fraction $\left(Z=\rho_{\mathrm{i}} / \rho_{\mathrm{n}}\right)$ is stirred by hydromagnetic turbulence in the ambipolar range, the turbulent velocity of the neutrals is higher in the most ionized regions, since they are better coupled to the ions $\left(v_{\mathrm{ni}}\right.$ is higher). This gives rise to a force, given by the average of the $v . \nabla v$ term, driving the neutrals out of the most ionized regions. By reaction the ions and magnetic flux are compressed in these regions, so that the initial ionization inhomogeneities are strongly amplified. This is expected to result in a condensation of the flux tubes, producing a filamentary structure. 
In order to study the efficiency of this non-linear mechanism, we simulated its behavior numerically (Franqueira et al., 2000) using simplified boundary conditions. With this first numerical experiment we confirmed the theoretical predictions made by Tagger et al. (1995).

In this contribution we present a step forward: a study of the relevance of this mechanism in the warm ionized component of the difuse interstellar medium (ISM). The ionization source of this warm ionized medium (WIM) is not completely understood; even if photoionization from early type stars seems to be the main source, additional heating sources are required (Reynolds et al., 2000). Minter and Spangler (1997) have shown that dissipation of hydromagnetic turbulence by ionneutral friction can play an important role as a source of heating. Indeed, large filamentary structures have been detected in $\mathrm{H} \alpha$ emission (Haffner et al., 1998), and density inhomogeneities have been observed in a wide range of scales (Minter and Spangler 1996).

\section{Simulation of the Ambipolar Filamentation in the WIM}

\subsection{THE NUMERICAL CODE}

Our code is based on the same general methods as the ZEUS-2D code (Stone and Norman, 1992). It solves the magnetohydrodynamic (MHD) equations describing a two-fluid (ions and neutrals) system on a cartesian staggered mesh using the method of finite differences with a time explicit, multistep (operator split) scheme (see Franqueira et al. (2000) for a complete description of the numerical code). We simplify the equations by assuming that all quantities are $x$-invariant, so only the perturbed current has a component along $x$, which is taken into account. Then, the problem becomes two-dimensional (in $y$ and $z$ directions).

\subsection{PARAMETERS: INITIAL AND BOUNDARY CONDITIONS}

Table I shows the WIM average parameters (from Minter and Spangler, 1996), and the spatial and time steps used in the calculations. We have normalized the parameters to the characteristic scales of the problem we are solving. Therefore we have taken as units the initial Alfvén velocity at $z=0, v_{\mathrm{A}}$, and the Alfvén time $t_{\mathrm{A}}$, defined as the time that takes such an Alfvén wave in crossing one wavelength $\lambda_{\mathrm{A}}$.

The gas is initially threaded by a constant magnetic field in $z$ direction that will be perturbed by transverse Alfvén waves excited at the footpoints of the magnetic field lines $(z=0)$. We assume initial equilibrium in a fluid stratified and supported by gravity, with ion and neutral densities decreasing sharply in $z$ direction (Figure 1, left). Initially the neutral density is independent of $y$, but the ion density shows a small enhancement in the $y$ direction (Figure 1, right). The magnetic field is 
TABLE I

Parameters used in the calculations: magnetic field, Alfvén velocity, sound velocity, perturbed velocity, neutral-ion collision frequency, ionization fraction, neutrals density, wave frequency, spatial and time steps

\begin{tabular}{lll}
\hline Parameter & Gaussian units & Normalized units \\
\hline$B$ & $8.5 \times 10^{-7} \mathrm{Gauss}$ & 0.52 \\
$v_{\mathrm{A}}$ & $20 \times 10^{5} \mathrm{~cm} / \mathrm{s}$ & 1 \\
$c_{\mathrm{s}}$ & $10 \times 10^{5} \mathrm{~cm} / \mathrm{s}$ & 0.5 \\
$v_{\mathrm{t}}$ & $7 \times 10^{5} \mathrm{~cm} / \mathrm{s}$ & 0.35 \\
$v_{\mathrm{ni}}$ & $1.0 \times 10^{-9} \mathrm{~Hz}$ & 7.2 \\
$Z$ & 0.8 & 0.8 \\
$\rho_{\mathrm{n}}$ & $1.0 \times 10^{-25} \mathrm{~g} / \mathrm{cm}^{3}$ & 0.15 \\
$\omega$ & $1.4 \times 10^{-10} \mathrm{~Hz}$ & 1 \\
$d y$ & $3.6 \times 10^{15} \mathrm{~cm}=0.00125 \mathrm{pc}$ & 0.25 \\
$d z$ & $5.04 \times 10^{15} \mathrm{~cm}=0.00175 \mathrm{pc}$ & 0.35 \\
$d t$ & $7.2 \times 10^{7} \mathrm{~s}=2.28$ years & 0.01 \\
\hline
\end{tabular}
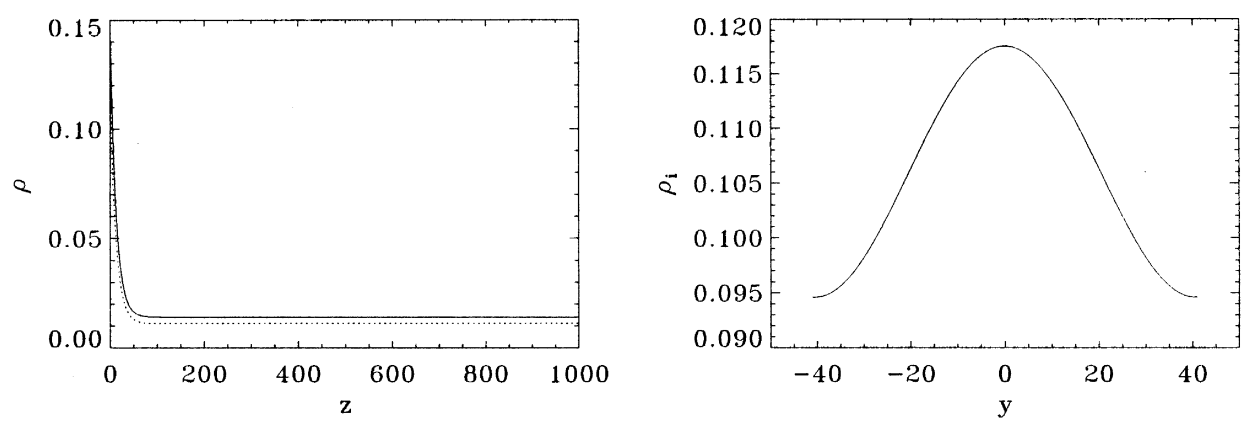

Figure 1. Left: Initial ions (dotted line) and neutrals (solid line) density profiles in the $z$ direction at $y=0$. Right: Initial ions density profile in the $y$ direction at $z=0$. We use the grid zone indices to scale the horizontal $(y)$ and vertical $(z)$ directions in all figures.

adjusted to ensure pressure equilibrium in the $y$ direction, that is, $\frac{\partial}{\partial y}\left(p_{\mathrm{i}}+\frac{B^{2}}{8 \pi}\right)=0$. The resulting initial ionization fraction $Z_{0}$ is constant over $z$ but shows a maximum at $y=0$ (Figure 2, left).

The boundary conditions are periodic in the $y$ direction. At $z=0$ we launch an Alfvén wave by giving the whole fluid (neutrals, ions and magnetic field lines) a motion in $y$ : a single periodic oscillation $v_{y}(y, 0)=v_{t} \cos (\omega t)$. This motion will propagate upward as an Alfvén wave. We chose a total length in $z$ direction such that the wave is heavily damped before it reaches the opposite boundary $\left(z_{\max }\right)$, where outflow boundary conditions are imposed.

The frequency of the wave was chosen so that it propagates without damping at the lowest part of the simulation grid $\left(\omega \ll v_{\mathrm{ni}}\right)$, but is strongly damped at higher 

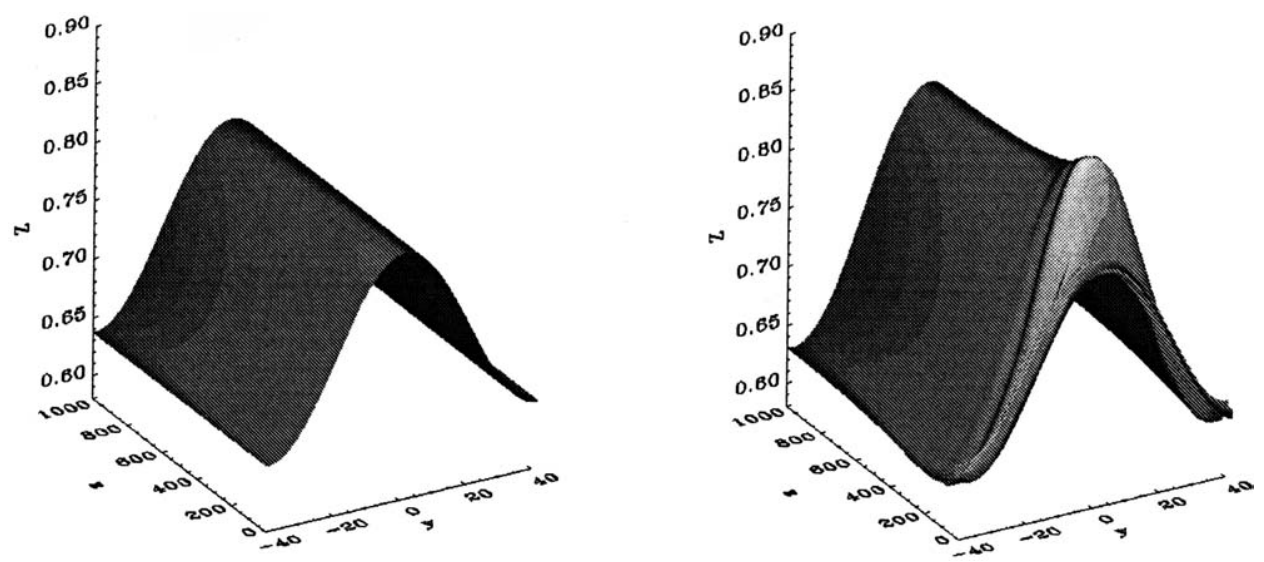

Figure 2. Left: Initial 2D distribution of the ionization fraction $Z=\rho_{\mathrm{i}} / \rho_{\mathrm{n}}$ in the calculation grid. Right: Spatial profile of the ionization fraction after $7.5 \times 10^{3} t_{\mathrm{A}}$.

altitudes in $z$, where $\rho_{i}$ (and thus $v_{\text {ni }}$ ) was taken to decrease sharply. Therefore the filamentation will occur at the altitude where the wave is damped (Tagger et al., 1995; Franqueira et al., 2000) and still retains a strong perturbed velocity.

\subsection{RESULTS}

We find an amplification of the contrast in $Z$ (Figures 2 and 3), from the altitude where the wave is damped. At the same region the $\rho_{\mathrm{i}}$ contrast along $y$ grows and the most ionized region shrinks (Figure 4, left). The neutrals are expelled from the most ionized regions (Figures 4, right), generating in the density profile in $y$ direction a "central" minimum. On the other hand, we are able to see (Figure 5, left) the velocity regime generated in $z$ direction (the direction of propagation of the wave) by the acceleration of material caused by ion-neutral friction and predicted
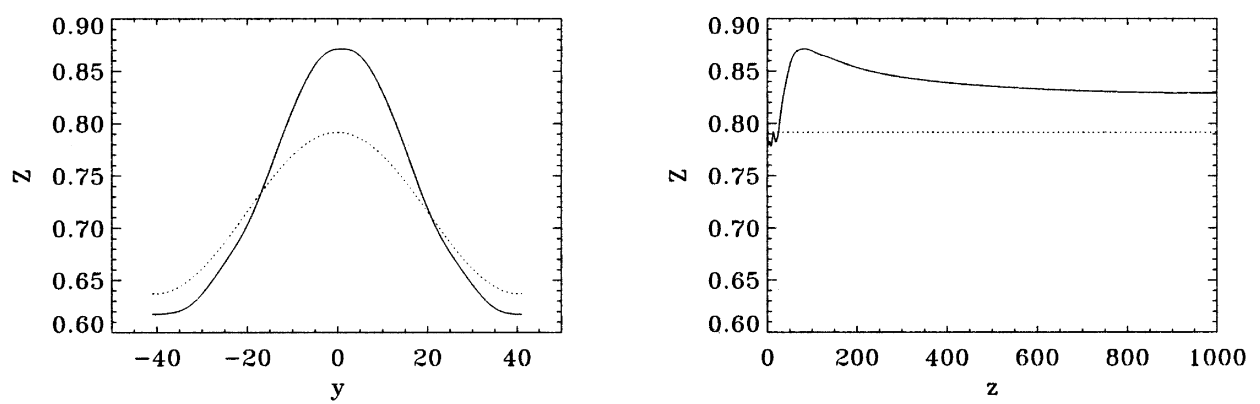

Figure 3. Ionization fraction profiles in $y$ direction (left) at the altitude where it reaches its maximum, and in $z$ direction (right) for $y=0$, after a $7500 t_{\mathrm{A}}$ calculation. Dotted lines show the initial values. 

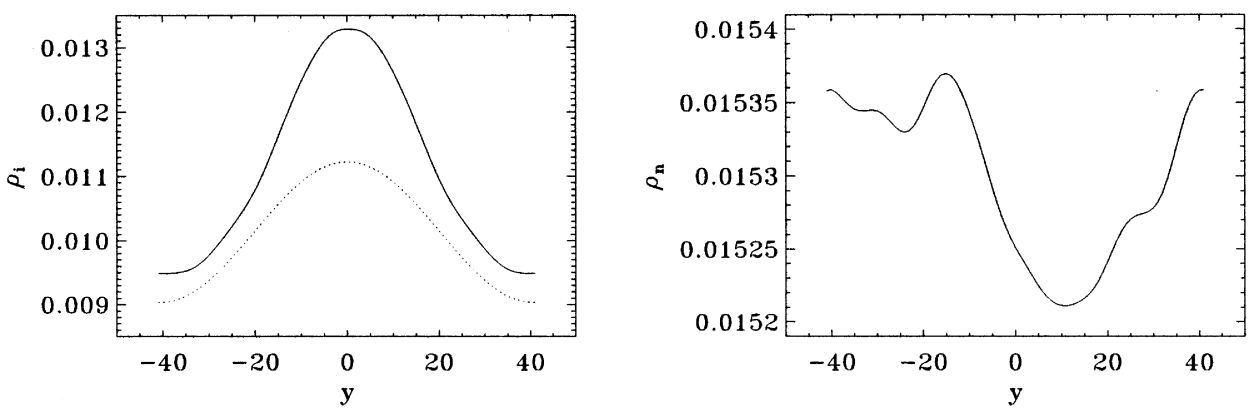

Figure 4. Ions (left) and neutrals (right) density profiles in $y$ direction at the altitude where the ionization fraction reaches its maximum after a $7500 t_{\mathrm{A}}$ calculation. Dotted lines show the initial values.

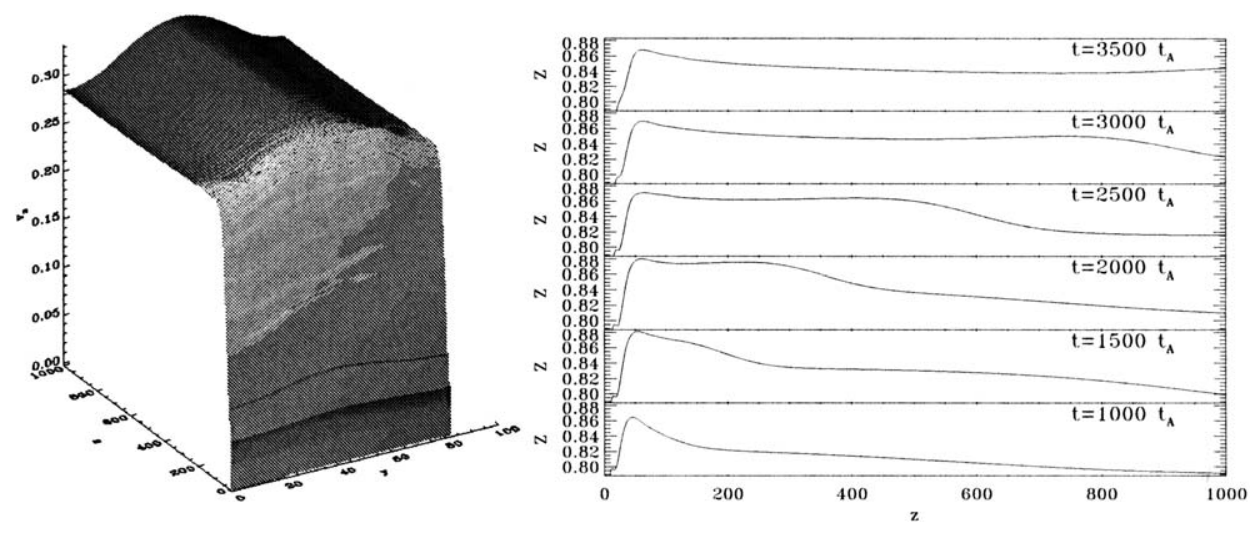

Figure 5. Left: 2D distribution of the z-component of the perturbed velocity (the same for ions and neutrals) at $t=7500 t_{\mathrm{A}}$. Right: Time evolution of the ionization fraction profile in $z$ direction at $y=0$.

by Haerendel (1992). As a consequence the profiles shown in Figures 3 (left) and 4 are transported in $z$ direction as shown in Figure 5 (right). Moreover, in Figure 5 (left) we find that the momentum transferred by the wave shows a maximum in the most ionized regions.

\section{Conclusions}

We find evidences of weak filamentation for the average conditions in the WIM. We also find momentum transfer in the direction of propagation of the wave caused by ion-neutral friction, that is more efficient in the most ionized regions, thus favouring the transport of ions.

The ambipolar filamentation results in an effective spatial separation of ions and neutrals at the altitude $z$ where both components decouple. The next step of our 
study will be to investigate the ambipolar filamentation process in molecular cloud conditions, where the turbulence is supersonic. We expect this mechanism will provide an additional pressure from the most ionized to the less ionized regions, efficiently separating the ions from the neutrals and favouring the gravitational collapse of the latter.

\section{References}

Franqueira, M., Tagger, M. and Gómez de Castro, A. I.: 2000, A\&A 357, 1143.

Haerendel, G.: 1992, Nat. 360, 241.

Haffner, L.M., Reynolds, R.J. and Tufte, S.L.: 1998, ApJ 501, L83.

Kulsrud, R. and Pearce, W.: 1969, ApJ 156, 445.

McIvor, I.: 1977, MNRAS 178, 85.

Mestel, L. and Spitzer, L.: 1956, MNRAS 116, 503.

Minter, A.H. and Spangler, S.R.: 1996, ApJ 458, 194.

Minter, A.H. and Spangler, S.R.: 1997, ApJ 485, 182.

Piddington, J.H.: 1956, MNRAS 116, 314.

Reynolds, R.J., Haffner, L.M. Tufte, S. L.: 2000, in: J. Arthur, N. Brickhouse, and J. Franco (eds.), Astrophysical plasmas: Codes, Models and Observations, Rev. Mex. Astron. Astrof. (Serie de conferencias) Vol. 9, p. 249.

Tagger, M., Falgarone, E. and Shukurov, A.: 1995, A\&A 229, 940. 